\title{
Deep Learning Enable Diagnostics and Prognostics of Machine Health Condition
}

\author{
Wo Jae Lee ${ }^{1}$ and John W. Sutherland ${ }^{2}$ \\ ${ }^{1,2}$ Purdue University, West Lafayette, IN, 47906, US \\ lee2465@purdue.edu \\ jwsuther@purdue.edu
}

\begin{abstract}
This paper presents an overview of the first author's research being conducted and future research plans for the rest of $\mathrm{PhD}$ career. The content of this paper was presented at the PHM 2019 Doctoral Symposium, which was a part of the program at the 11th Annual Conference of the Prognostic and Health Management Society held in Scottsdale, Arizona from September 21-26, 2019. The paper covers the development and application of data-driven approaches to machine health management.
\end{abstract}

\section{INTRODUCTION}

In the 21 st century, Industry 4.0 has attained significant momentum with the advancement in technologies such as smart sensor, AI, and IoT). Those technologies are transforming a manually operated factory into a highly automated plant, and enable to response in real time to changing conditions (Lee, Mendis, \& Sutherland, 2019). This new trend is expected to improve the current maintenance practices significantly in manufacturing plants.

In the field of machine condition monitoring research, three different models were often used to enhance machine reliability. The models are 1) a physics-based model, a knowledge-based model, and a data-driven model (Lee, $\mathrm{Wu}$, Yun, Kim, Jun, \& Sutherland, 2019). A physics-based model shows a fairly good performance on reflecting the condition of the system, because the model is based on the accurate mathematical relation tied to physical processes. However, it is challenging to establish a good physical model for complex manufacturing equipment. A knowledge-based model (e.g., expert systems) attempts to solves a specific domain problem using expert knowledge and heuristic rules. In this method, translating a domain knowledge into rules is challenging and the system could not handle with new situations properly.

Wo Jae Lee et al. This is an open-access article distributed under the terms of the Creative Commons Attribution 3.0 United States License, which permits unrestricted use, distribution, and reproduction in any medium, provided the original author and source are credited.
Lastly, a data-driven model has obtained a significant popularity due to an advancement in the computing performance. The challenge of the data-driven model is it requires a huge amount of labelled data for model training and validation.

In Industry 4.0 era, many emerging technologies (e.g., artificial intelligence, IoT, and smart sensor) will significantly affect the current maintenance practice. Thus, the author is not only researching potential benefits of the emerging technologies in the maintenance practice, but also applying the technologies into real machinery system to experimentally demonstrate the benefits.

\section{GUIDELINES EXPECTED CONTRIBUTION}

As mentioned above, a data-driven model has gained significant popularity because the method is able to handle various types of data and discover hidden connections in the large-scale data. Thus, this method may be a useful tool to identify a health condition of manufacturing equipment using sensor signals; however, the model may work well only for a specific operation setting where training dataset are available. Also, it may become harder to predict the remaining useful life of a component if many rolling elements (e.g., bearing, shaft, and rotor) are working together e.g., motor and pump. Thus, crucial to a successful condition monitoring is how to monitor in-service equipment and how to extract meaningful information from a large amount of data collected from the machine/process under dynamic environment.

The author's research topics is a development and application of robust deep learning models for the machine health diagnostics and prognostics, i.e., from sensor data to maintenance task. The robust model means a model can predict the machine (e.g., motor) health state under dynamic situation (e.g., varying load and RPM). In the method, a deep learning model is designed to be trained to learn key features for the degradation of each machine component regardless of varying condition; this is called invariant learning. Also, the proposed model will be customized to be specifically applied in the machine maintenance, i.e., the output of model is 
remaining useful life of each component in equipment, and this information will be used for maintenance scheduling.

The work is currently undergoing, and the developed method is expected to support decision making in maintenance area. In other words, as shown in Fig. 1, the maintenance schedule can be optimized by quantifying the remaining life of each component, and consequently reduce maintenance cost by simultaneously decreasing machine downtime and repair cost.

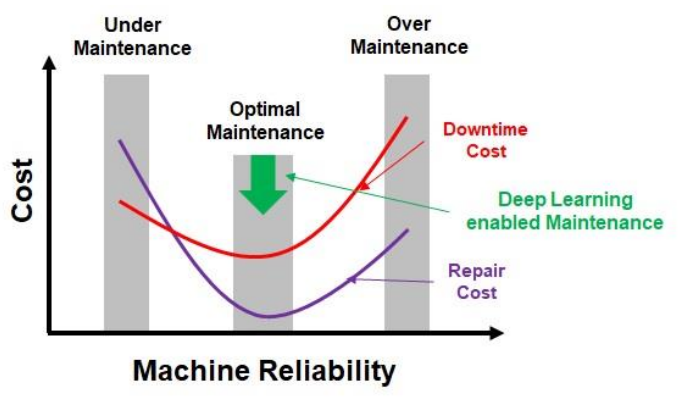

Figure 1. Cost vs machine reliability.

\section{RESEARCH PLAN}

In order to develop a robust model, and then demonstrate an effectiveness of proposed method, the author's research plan for next three years is as follows; 1) design and develop of a robust deep learning architecture (e.g., invariant representation learning) specifically applicable for the machine health diagnostics and prognostics, 2) demonstrate the effectiveness of the proposed method both in a lab-scale machine testbed and in a manufacturing plant, and 3) optimize maintenance schedule using deep learning enabled $\mathrm{PdM}$ method and quantify the economic benefit.

\subsection{Work Performed}

The work being conducted by the author can be divided into two sections: 1) setting up a lab-scale motor testbed in which various fault conditions (e.g., broken gear tooth, mechanical looseness, and misalignment) can be configured as shown in Figure 2 and 2) collaborating with industry (i.e., manufacturing company) to deploy wireless vibration sensor in the manufacturing plant as shown in Figure 4. As mentioned before, the goal of author's research is to prove the effectiveness of the proposed deep learning method in both lab and real manufacturing environment.
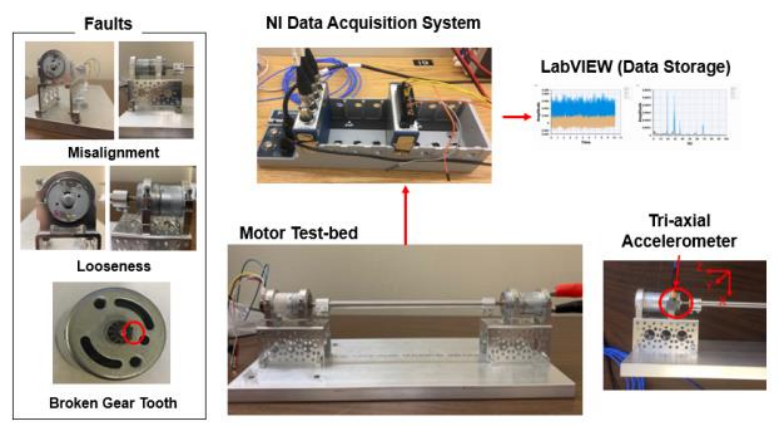

Figure 2. Lab-scale motor testbed.

Frist, a lab-scale motor testbed was set up to introduce several fault modes in the motor system. Tri-axial acceleration signals were collected during the experiment, and eight different conditions including baseline (normal condition) were introduced; details regarding the experiment set up and procedure can be found in Lee, Wu, Huang, \& Sutherland (2019). The signals collected during the experiment were preprocessed, and the processed signals were used as training and testing dataset for deep learning and machine learning algorithms. Figure 3 compares the prediction accuracies of several classification algorithms; four CNN models (Simple, GoogLeNet, Alex Net, ResNet50) and machine learning models including ANN, SVM, DT, KNN, and QDA. In the figure, Type I, II, and III means the different training approaches, i.e., increasing the number of training dataset. The result shows that the deep learning methods outperform the machine learning methods for classification task when using the tri-axial acceleration signals obtained from the motor testbed. Future work regarding the motor test bed will be explained in section 3.2.

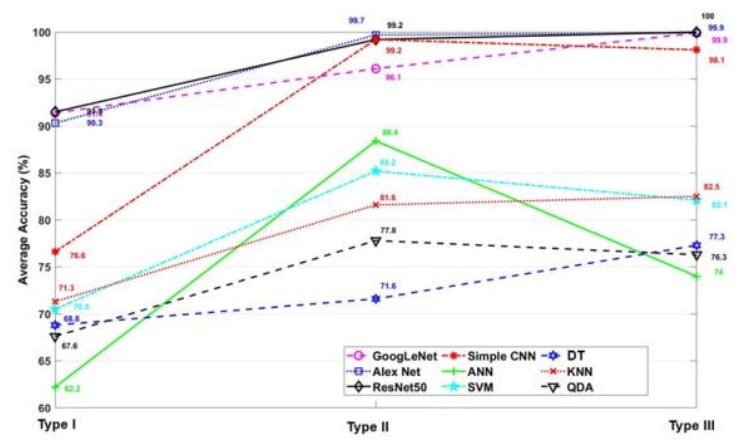

Figure 3. Prediction accuracy comparison between deep learning and machine learning models.

Second, the commercial wireless tri-axial acceleration and temperature sensors manufactured by Bluvision and Fluke were deployed in a manufacturing plant near Purdue University as shown in Figure 4. The overview of the data transmission (from sensor to the cloud system) are explained 
in Figure 5, and tri-axial acceleration data has been collected for past 10 months.

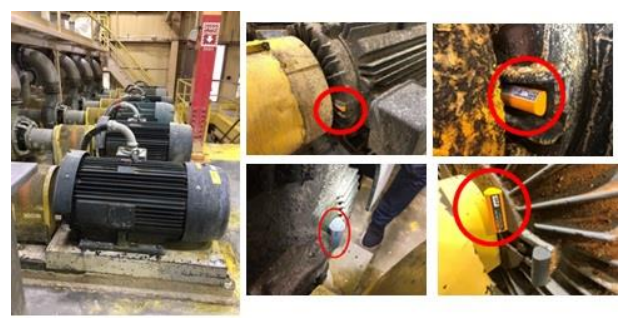

Figure 4. Deployment of wireless vibration and temperature sensors in manufacturing plant.

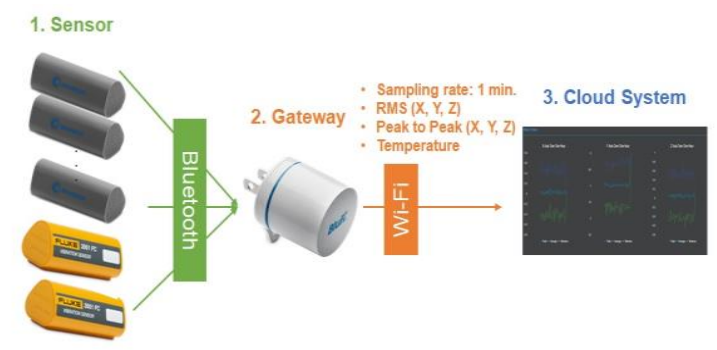

Figure 5. Data collection procedure: from wireless sensors to cloud system.

Since the data from the manufacturing field were mostly obtained while the motors were normal (no critical failure happens so far), an unsupervised learning method were employed to monitor any future abnormal behavior of the motors being monitored. As shown in Figure 6, data obtained during the abnormal condition (e.g., power outage) were visualized using kernel principal component analysis (KPCA) on the left side, and KPCA-modified data were represented in the control charts for Hotelling $T^{2}$ and $Q$ statistic with the control limit of $99 \%$ (Lee, Mendis, Triebe, \& Sutherland, 2019); this is one example using the data collected from one motor.

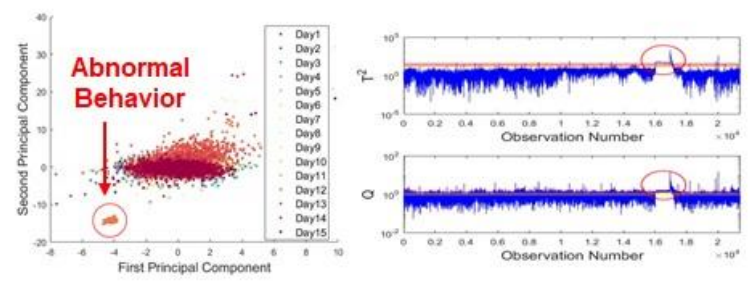

Figure 6. Abnormality Monitoring System.

\subsection{Remaining Work}

From the previous work, the deep learning models show high prediction accuracy in the experimental setting where motor load and RPM were set to constant. However, the model may not work well in the dynamic environment (e.g., varying
RPM and load) since the model were trained by the data obtained under the specific operation setting. Thus, the author will study a new deep learning architecture, which will be invariant to RPM and load when predicting the machine condition, i.e., design a deep learning architecture specifically applicable for machine diagnostics and prognostics.

Once a new deep learning architecture is prepared, it will be tested in both lab testbed and manufacturing plant. Several sets of run to failure experiment will be conducted using the different size of motor to collect data. The author is closely working with several maintenance engineers in the manufacturing plant, thus, ultimately, the key factors under the deep learning enabled maintenance practice (e.g., down time cost, repair cost, and maintenance scheduling) will be quantified.

\section{Conclusion}

In this paper, the author's on-going research works and future research plan as well as the research motivation and expected contribution are summarized. The goal of the author's research is to develop a robust deep learning architecture, which is able to estimate machine health condition under dynamic environment, and optimize maintenance schedule based on the proposed deep learning model, i.e., from sensor data to maintenance schedule.

\section{ACKNOWLEDGEMENT}

This work is supported by the Wabash Heartland Innovation Network (WHIN). Any opinions, findings, conclusions, and/or recommendations expressed are those of the authors and do not necessarily reflect the view of the WHIN.

\section{REFERENCES}

Lee, W. J., Wu, H., Yun, H., Kim, H., Jun, M. B. G., \& Sutherland, J. W. (2019). Predictive maintenance of machine tool systems using artificial intelligence techniques applied to machine condition data. Procedia CIRP, vol. 80, pp. 506-511. doi:10.1016/j.procir.2018. 12.019

Lee, W. J., Mendis, G. P., \& Sutherland, J. (2019). Development of an intelligent tool condition monitoring system to identify manufacturing tradeoffs and optimal machining conditions. Procedia Manufacturing, vol. 33, pp. 256-263. doi:10.1016/j.promfg.2019.04.031

Lee, W. J., Wu, H., Huang, A., \& Sutherland, J. (2019). Learning via Acceleration Spectrograms of DC Motor System with Application to Condition Monitoring. The International Journal of Advanced Manufacturing Technology.

Lee, W. J., Mendis, G. P., Triebe, M., \& Sutherland, J. (2019). Monitoring of a Machining Process using Kernel Principal Component Analysis and Kernel Density Estimation. Journal of Intelligent Manufacturing. 


\section{BIOGRAPHIES}

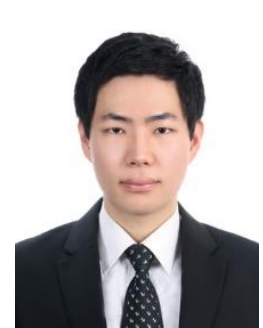

Wo Jae Lee is a Ph.D. student under the supervision of Prof. John W. Sutherland at Purdue University. Wo Jae received a Bachelor's degree from Korea University, Seoul, Korea. He is currently a research assistant in the Laboratory for Sustainable Manufacturing, and his research interests include smart and sustainable manufacturing, deep learning, and intelligent maintenance systems.

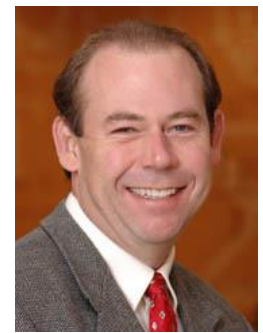

John W. Sutherland is the Fehsenfeld Family Head of Environmental and Ecological Engineering (EEE) at Purdue University. He received his Ph.D. degree in mechanical engineering from the University of Illinois at UrbanaChampaign, and is a fellow of SME, ASME, and CIRP. His research focuses on the application of sustainability principles to design, manufacturing, and other industrial issues. 